\title{
Transformaciones fonológicas, morfológicas y semánticas de los apellidos aimaras: Quispe, Mamani, Colque, Choque y Condori
}

\section{Phonological, Morphological and Semantic Transformations of the Aymara Surnames: Quispe, Mamani, Colque, Choque And Condori}

\author{
Milusca T. Arocutipa Saira \\ Universidad Nacional del Altiplano, Puno, Perú \\ https://orcid.org/oooo-0002-6381-842X \\ milusca.arocutipa@gmail.com \\ Elizabeth Castrillo Yavar \\ Universidad Nacional del Altiplano, Puno, Perú \\ https://orcid.org/oooo-00o2-8466-6330 \\ ruthelizabeth.7185@gmail.com
}

\author{
Carmen J. Mamani Paucar \\ Universidad Nacional del Altiplano, Puno, Perú \\ https://orcid.org/oooo-0002-7300-8500 \\ carmenmamani.puk@gmail.com \\ Vilma Ramos Maque \\ Universidad Nacional del Altiplano, Puno, Perú \\ https://orcid.org/oooo-0oo2-6884-5750 \\ ramos.maque.v@gmail.com
}

\begin{abstract}
Resumen
En algún momento de sus vidas las personas se preguntan sobre el significado que tienen sus nombres y apellidos. No obstante, pocos logran conocerlo, ya que muchos de estos provienen de una lengua originaria; los cuales, con el tiempo perdieron su significado y originalidad, debido al contacto entre lenguas y culturas. Por ello, en el presente artículo se analiza desde el enfoque antroponímico las transformaciones que se han dado a nivel fonológico, semántico o morfológico en los apellidos aimaras; para ello, se utiliza el método de documentación escrita, puesto que se recurre a investigaciones realizadas sobre los apellidos aimaras. En ese sentido, el artículo concluye que los apellidos sufrieron transformaciones fonológicas, morfológicas y semánticas, debido a la sustitución de fonemas oclusivos por fonemas simples, la sustitución de fonemas aimaras por fonemas del castellano y la adición de fonemas, lo que ocasionó la pérdida de su significado y originalidad.
\end{abstract}

Palabras claves: antroponimia, aimara, fonología, morfología y semántica

\begin{abstract}
At some point in their lives, people wonder about the meaning of their first and last names. However, few get to know it, since many of these come from an original language; which, over time, lost their meaning and originality, due to contact between languages and cultures. Therefore, this article analyzes from the anthroponymic approach the transformations that have occurred at the phonological, semantic or morphological level in Aymara surnames; For this, the written documentation method is used, since it uses] research carried out on Aymara surnames. In this sense, the article concludes that the surnames that underwent phonological, morphological and semantic transformations, due to the substitution of stop phonemes for simple phonemes, the substitution of Aymara phonemes for Spanish phonemes and the addition of phonemes, which caused the loss of its meaning and originality.
\end{abstract}

Keywords: anthroponymy, Aymara, phonology, morphology and semantics 
Transformaciones fonológicas, morfológicas y semánticas de los apellidos aimaras... Milusca T. Arocutipa, Elizabeth Castrillo, Carmen J. Mamani y Vilma Ramos

\section{Introducción}

Los apellidos fueron representaciones de la identidad cultural de las personas, los cuales sufrieron transformaciones a nivel fonológico, morfológico y semántico desde la invasión de los españoles al Tahuantinsuyo; puesto que muchos apellidos originarios perdieron su significado original.

En la actualidad, existe la antroponimia, rama de la lingüística encargada de realizar estudios acerca de los nombres y apellidos, al respecto RENIEC (2012) indica que el nombre «es la palabra o grupo de palabras que sirve para designar a una persona distinguiéndose de los demás» (p. 17). Esta disciplina contribuye a rescatar significados de nombres y apellidos provenientes de lenguas originarias, y ayuda a dar cuenta que la mayoría de los apellidos se originaron del propio entorno cultural y desde su cosmovisión. En la misma línea, Guerin, citado en Condori (2007), menciona que:

El significado de los apellidos responde, principalmente, a dos tipos de procedencia. Los apellidos procedentes de nombres propios (patronímicos) y los apellidos procedentes de un lugar. También existen los apellidos que se derivan de oficios, apodos, advocaciones religiosas, plantas y animales, rasgos físicos y expresiones, etc. (p. 56)

Lo mencionado por el autor indica que en los apellidos existen dos tipos de procedencia: primero, apellidos provenientes de abuelos a padres, padres a hijos y segundo, apellidos procedentes de lugares, lo cual muestra la presencia de apellidos que se originan del entorno. Asimismo, señala que existen apellidos que se derivan de los oficios en que las personas se desempeñan o de rasgos físicos.

Respecto a los estudios realizados acerca de la antroponimia quechua y aimara en específico, existen pocas investigaciones; por ejemplo Condori (2007), en su tesis denominada Estudio diglósico de la antroponimia en la cultura aymara, realiza un estudio de la antroponimia y la diglosia del idioma aimara para evitar los cambios de apellidos por la influencia del castellano de la segunda sección de la provincia Pacajes - Caquiaviri, donde da a conocer de manera detallada los cambios que se dieron en los aspectos fonológicos, morfológicos y semánticos de apellidos aimaras para la pérdida de sus significados. Dicho autor, para dar cuenta de los motivos sociales por las que los apellidos han desvirtuado su significado original, cita a De Lucca, quien señala que: 
Transformaciones fonológicas, morfológicas y semánticas de los apellidos aimaras... Milusca T. Arocutipa, Elizabeth Castrillo, Carmen J. Mamani y Vilma Ramos

la opresión y dominio que ejerce la lengua castellana desde la conquista. Donde el significado de los apellidos de indiscutible origen aymara ha sido desvirtuados y han perdido toda carga semántica que ellas tenían en un principio porque fueron avasallados por el remetimiento de poder del castellano (diglosia), y sometidos de acuerdo a las estructuras del idioma oficial y de prestigio. (Condori, 2007, p. 28)

El autor señala que los apellidos de origen aimara perdieron su originalidad, debido a que fueron sometidos a la estructura de la lengua castellana; ya que esta última ejercía un poder y dominio sobre las culturas y lenguas invadidas por los españoles. Por ende, el presente artículo de investigación tiene como objetivo analizar desde el enfoque antroponímico los cambios morfológicos, semánticos y fonológicos que se dieron en algunos apellidos aimaras; así como los ejemplos a continuación, «[...] Quispe se tornará Quisberg, los Muruchi será Marach, los Hiuthuya serán Bedoya y los Pakara se convertirán en Flores» (Pérez-Ruiz, 2000, p. 78). Lo señalado, da cuenta de los cambios que sufrieron dichos apellidos, algunos manteniendo su significado y otras cambiándolas totalmente.

El artículo realiza un estudio acerca de los apellidos aimaras, el cual está compuesto por cinco partes: primero, la introducción, en la cual se desarrolla un resumen del artículo; segundo, el marco teórico, donde se desarrolla el concepto de la antroponimia, concepto de la fonología, morfología y semántica, y el concepto de lengua aimara; tercero, la metodología del artículo; cuarto, el análisis de los apellidos Colque, Mamani, Quispe, Choque y Condori, y por último la conclusión.

\section{Marco teórico}

\subsection{Antroponimia}

La antroponimia es una disciplina que estudia la etimología y el significado de los nombres de las personas, al respecto el Registro Nacional de Identificación y Estado Civil (RENIEC, 2012), en el libro denominado Introducción a un tesoro de nombres quechuas en Apurímac, señala que la antroponimia «es la disciplina cuyo objeto de estudio son los sistemas de denominación de cada cultura y los nombres propios de las personas que dichos sistemas generan» (p. 13). Por lo señalado, afirma que la antroponimia estudia nombres de cada contexto o territorio, y nombres de los sujetos procedentes de padres o madres. En misma línea, la misma instancia, RENIEC (2015), en su texto llamado Tesoro de nombres Jaqaru Shumay 
Transformaciones fonológicas, morfológicas y semánticas de los apellidos aimaras... Milusca T. Arocutipa, Elizabeth Castrillo, Carmen J. Mamani y Vilma Ramos

shutxkuna Jaqarna, señala que la antroponimia «es la rama de la onomástica que estudia la etimología e historia de los nombres propios de las personas» (p. 13). Es decir, estudia la procedencia o raíz de los nombres propios.

Asimismo, Condori (2007) en su tesis denominado Estudio diglósico de la antroponimia en la cultura aymara, indica que la antroponimia conforma los nombres de pila, los apellidos, así como los apodos y seudónimos: primero, los nombres de pila tenían origen por influencias cristianas religiosas, pero hoy tienen origen en los meses, la flora, los lugares todo cuanto se encuentre en el entorno de las personas. Segundo, los apellidos provienen de oficios, ocupaciones o lugares de las cuales se transportan de generación en generación. De la misma forma, los apodos surgen de los defectos o cualidades físicos personales. Finalmente, los sobrenombres se originan de actitudes y comportamientos de una persona. Lo señalado por el autor tiene coincidencias con la cultura aimara, debido a que los apellidos se originan de los elementos de la misma naturaleza en la que conviven.

En la misma línea, RENIEC (2012), citado en Jacinto (2009), señala que «los antropónimos son los nombres de pila, los apellidos (patronímicos $=$ padres o matronímicos $=$ madres) así como de los sobrenombres (apodos o alias) y de los nombres diminutivos» (p. 46). Por lo tanto, es posible decir que la antroponimia no solo estudia los significados de los nombres propios de las personas, sino también sus apellidos provenientes de padres y madres, asimismo, los sobrenombres que existen dentro de una lengua. «En un sentido estricto, forma parte de la lingüística que estudia las propiedades formales funcionales o léxico-semánticas de los antropónimos» (Condori, 2007, p. 22). En ese entender, la comprensión de este concepto en este artículo, ayudará a abordar los significados, los aspectos léxico-semánticos de los apellidos aimaras y las transformaciones que han sufrido.

\subsection{La fonología, morfología y semántica}

\subsubsection{Fonología}

La fonología es una «ciencia que estudia los sonidos del lenguaje, desde el punto de vista de su función en el sistema de la comunicación lingüística» (Apaza, citado por Sonco, 2015, p. 18). Es decir, estudia los sonidos de las lenguas o formas de pronunciación de los hablantes. Asimismo, Pennington, citado y traducido por Sonco (2015), indica que:

224 Lengua y Sociedad. Revista de Lingüística Teórica y Aplicada 
Transformaciones fonológicas, morfológicas y semánticas de los apellidos aimaras... Milusca T. Arocutipa, Elizabeth Castrillo, Carmen J. Mamani y Vilma Ramos

La fonología es una descripción de los sonidos [...]. Más correctamente, podemos ver a la fonología [...] como modelo de pronunciación, como el estudio de los sonidos de lenguas o formas de pronunciación de los hablantes. (p. 1)

En ese sentido, el autor señala tres aspectos primordiales que realiza la fonología: primero, hace una descripción de los sonidos de una determinada lengua; segundo, la relación que existe entre estos sonidos; y tercero, el contraste que se realiza uno con el otro. Es así que la fonología se encarga de realizar investigaciones sobre los sonidos que tiene una lengua y las formas que existen en sus pronunciaciones.

\subsubsection{Morfología}

La morfología es una rama de la lingüística que viene del griego [morph] ma y logía: tratado= forma y [logía] = tratado, esta se encarga de realizar una investigación acerca de la estructura interna de la palabra para luego ordenarlos en unidades (Laura, 2014). En ese sentido, la morfología da un sentido a la estructura de las palabras, a fin de que pueda permitir hacer un orden minucioso o de manera detallada en cada palabra. De este modo, la morfología:

[...] es la parte de la gramática que estudia la estructura y formación de palabras y las variaciones [...] que experimentan en su uso, para expresar como unidades léxicas diferentes significados o distintas funciones que asume en la oración, según su relación con otras palabras de la emisión. (Orellana, 2007, p. 24)

Por lo señalado, se puede decir que la morfología se encarga de estudiar tres partes fundamentales de la gramática: primero, la estructura, el orden de las palabras; segundo, formación de las palabras y tercero las variaciones que existen en estas palabras. No obstante, Laura (2014) menciona que existen dos categorías de estudio; primero, la morfología flexiva estudia las variaciones que existe en cuanto a género y número, y segundo, la morfología léxica estudia las posibilidades para formar nuevas palabras por derivación o composición de una determinada lengua. Por lo mencionado por la autora, es posible decir que la morfología flexiva posibilita el estudio de cambios que se producen a nivel morfológico. En esa línea, dicho concepto contribuirá para explicitar los cambios morfológicos que se han dado en algunos apellidos aimaras. 
Transformaciones fonológicas, morfológicas y semánticas de los apellidos aimaras... Milusca T. Arocutipa, Elizabeth Castrillo, Carmen J. Mamani y Vilma Ramos

\subsubsection{Semántica}

La semántica dentro del campo de la lingüística estudia el significado de las palabras de una lengua. Al respecto, Laura (2014) señala que el estudio de la semántica permite conocer, por un lado, el significado de las palabras y, por otro lado, los problemas que se suscitan sobre los significados. Es decir, contribuye a recopilar información sobre lo que significa una o más palabras de una determinada lengua. En ese sentido, se considera que la semántica contribuirá para la comprensión de los significados de los apellidos aimaras.

Sin embargo, es preciso mencionar que las palabras de las lenguas andinas, como el aimara, han sufrido cambios y transformaciones al ser castellanizadas, a ello se llama cambio léxico-semántico, ya que «[...] es el más propenso al cambio porque refleja de forma muy clara las modificaciones y transformaciones como, por ejemplo; QUMPI [qompi] a COMPI. Propias de la evolución cultural de las culturas humanas» (Laura, 2014, p. 32). Por lo señalado, es posible decir que dicha evolución cultural, implícitamente también se debe a factores sociales como el contacto entre lenguas, las situaciones de discriminación y la alienación cultural; lo que repercute en la transformación semántica de las palabras y posteriormente en el desplazamiento de las lenguas minoritarias. En esa línea, este artículo recurrirá al concepto y ejemplo descrito para dar a conocer los cambios semánticos que se dieron en algunos apellidos aimaras.

\subsection{Lengua aimara}

Los apellidos que se abordan en este artículo son de origen aimara. Dicha lengua, a nivel geográfico es hablada en Perú (Puno, Tacna y Moquegua), Bolivia (La Paz, Oruro y Cochabamba), también en el norte de Chile (Arica, Iquique y Antofagasta) y en Argentina (Salta y Jujuy). No obstante, antiguamente se hablaba en las colonias de Chachapoyas, Alto Marañón, y Áncash.

Actualmente, es la segunda lengua originaria con mayor población de hablantes a nivel nacional siendo «[...] uno de los idiomas oficiales con mayor cantidad de hablantes, que varían entre 300 ooo y 600 ooo en el Perú, y 1400 ooo y 160000 [sic] en Bolivia» (Lovón, 2019, p. 59). Como señala el autor, la lengua aimara es uno de los idiomas oficiales que tienen una mayor cantidad de hablantes entre los países mencionados.

La palabra aimara a lo largo de los años sufrió cambios, según Cerrón-Palomino, citado por el RENIEC (2018), indica que la palabra originaria fue aymarawi, el cual

226 Lengua y Sociedad. Revista de Lingüística Teórica y Aplicada 
Transformaciones fonológicas, morfológicas y semánticas de los apellidos aimaras... Milusca T. Arocutipa, Elizabeth Castrillo, Carmen J. Mamani y Vilma Ramos

tuvo un proceso evolutivo pasando a aymaray, posteriormente aymara, luego aymaraes y finalmente aimara.

\subsection{Antroponimia aimara}

Los aimaras antes de la invasión española generalmente se identificaban con sus apellidos más que con sus nombres por familia, las que actualmente son considerados como apellidos (Cuaquira, 2014). Teniendo en cuenta que, en estas se lleva una identidad cultural y un significado especial; así, por ejemplo; «Atawallpa (ataw- viril y hallpa-tierra dulce negra), Coya Rithi (coya-Luna, rithi-fulgor), [...], Rumiñahui (rumi-piedra, ñahui-ojo "quechua"), Willkapampa (willka-águila, pampa-llanura), Yaguarsongo (yaguar-sangre, songo-valle), [...], Coya (luna)» (Coaquira, 2014, p. 7). Los apellidos de los habitantes del Tahuantinsuyo estaban estrechamente relacionados con la cosmovisión de la cultura, debido a que se originan de elementos de la naturaleza como luna, piedra, águila, llanura, entre otros. Sin embargo, muchos de esos apellidos originarios mencionados sufrieron alienación de su identidad, puesto que muchos incorporaron a sus nombres préstamos de apellidos españoles, así como el nombre de sus patrones; de esta manera, quedó en segundo plano sus nombres originarios; así por ejemplo, «Julián Ayala, [...], Manuel Pacas, Tomás Katari (cacique quechua), [...], José Gabriel Condorkanqui (cacique de Tuyansuca), JuliánApaza (Dirigente aymara), Curasa Ilavi, Nicolás Katari, Miguel Condorkanqui (padre de José Grabiel Condorkanqui), Micaela Bastidas Ph"uyuqhawa (esposa de Tupaj Amaru), [...]» (Coaquira, 2014, p. 8). Es por eso que los varones y mujeres portan en la actualidad apellidos de denominación española. No obstante, es preciso señalar que en la actualidad los apellidos son originarios de las lenguas quechua, aimara o puquina, son traducidos al castellano.

Asimismo, fueron cambiados a nivel léxico y gramatical por voluntad propia o por obligación, puesto que hace años atrás la RENIEC no permitía el registro de nombres ni apellidos originarios, ya que los funcionarios no sabían la lengua del hablante. Es por ello, que los trabajadores registraban los nombres y apellidos desde el sistema fonológico y gramatical del castellano. No obstante, en pleno siglo XXI dicha situación aún persiste por parte de la RENIEC, es por eso que muy pocos logran registrarse con nombres originarios.

Desde la mirada lingüística, en la actualidad los apellidos aimaras no guardan relación entre el significado y el significante. Ya que la mayoría de los apellidos han perdido su significado original. Sin embargo, aún existen apellidos que mantienen 
Transformaciones fonológicas, morfológicas y semánticas de los apellidos aimaras... Milusca T. Arocutipa, Elizabeth Castrillo, Carmen J. Mamani y Vilma Ramos

su significado y significante como: Tintaya, Sarpi o Yujra, pero las personas que portan esos apellidos desconocen su significado. También, existen apellidos que cambiaron su forma y significante como los apellidos Villka a Villca, Yujra a Yucra y Azuaya a Zaballa (Cuaquira, 2014). Por lo mencionado, es posible decir que las personas portadoras de apellidos originarios de lenguas como el aimara desconocen su significado de su apellido, debido a que con el tiempo han ido cambiando semánticamente y junto a ello han ido perdiendo su significado original.

En la actualidad, varias personas que tenían apellidos aimaras optaron por cambiarlas traduciéndolas literalmente al castellano, como, por ejemplo: panqara - Flor o Flores, paxsi - Luna, k'achita - Hermosa, janq'u - Blanco, qullqi - Plata. Sin embargo, todavía existen apellidos en aimara como: Ch’uqi, Katari, Sariri o Mamani, los cuales no tienen una traducción fija en castellano (Coaquira, 2014). De esta manera, muchos apellidos al ser traducidos del aimara al castellano han sido desplazados de su significado que tenían en la lengua aimara.

\section{Metodología}

El presente artículo analiza cinco apellidos aimaras, debido a que es una investigación cualitativa, ya que ésta, se encarga de interpretar información a través de recursos comunicativos con el propósito de indagar su significado y origen de procedencia. La metodología de investigación proporciona profundidad a los datos, dispersión, riqueza interpretativa, contextualización del ambiente o entorno, detalles y experiencias únicas. Asimismo, aporta un punto de vista «fresco, natural y holístico de los fenómenos, así como flexibilidad» (Hernández, et al., 2014, p. 16). Por ende, es un instrumento que guía y orienta el desarrollo de una investigación científica. Interpreta y analiza los datos de una investigación cualitativa, dando a conocer detalladamente el entorno de dicha investigación. De igual forma, los investigadores indican que los métodos siguen una serie de páginas, y por ser natural estas a razón de los años pueden actualizarse o cambiar, también se puede abarcar de manera general amplificando según al avance se puede dar una serie de observaciones, como ver la obtención de resultados que se propone o adaptar esta metodología.

Para ello, se tomó como método de documentación escrita acerca del estudio de los apellidos aimaras: Qullqi, Mamani, Qhispi, Ch'uqi y Kunturi. Teniendo en cuenta que, la metodología significa ciencia del método, y este está relacionado con las herramientas de recolección de datos (Gómez, Jean-Pierre y Alzate, 
Transformaciones fonológicas, morfológicas y semánticas de los apellidos aimaras... Milusca T. Arocutipa, Elizabeth Castrillo, Carmen J. Mamani y Vilma Ramos

2010), por eso, se tomó en cuenta estos cinco apellidos aimaras porque son los más comunes a nivel nacional.

La muestra poblacional del estudio cualitativo son apellidos originarios de la cultura aimara, ya que, estos apellidos en su mayoría son comunes en nuestro país. Para ello, se ha utilizado el libro Tesoros de nombres aymaras elaborado por el RENIEC y el ¿Nombres y apellidos? El sistema nominativo aymara sacaca, siglo xvir de Medinaceli. Asimismo, se usó una tesis denominada Estudio diglósico de la antroponimia en la cultura aymara desarrollado por Condori, los cuales se usarán para el análisis de los significados de transformaciones fonológicas, morfológicas y semánticas de apellidos aimaras.

\section{Análisis de los apellidos: Colque, Mamani, Quispe, Choque y Condori}

\subsection{Qullqi - Colque}

La refonologización o castellanización del apellido Qullqi:

Tabla 1. Transformación del apellido Colque

\begin{tabular}{l|l|l}
\hline Forma original & Forma actual & Significado \\
\hline Qullqi & Colque & $\begin{array}{l}\text { La palabra significa } \\
\text { metal o plata. }\end{array}$ \\
\hline
\end{tabular}

Fuente. Elaboración propia.

Coaquira (2014) manifiesta, que el proceso de refonologización ocasionó una serie de consecuencias < fonemas del idioma castellano, como en los casos: «Paqu $>$ Paco», «Qantuta <Cantuta», «Qullqi > Colque».

Al realizar la acomodación del sonido de los apellidos aimaras dentro del sistema fonológico castellano se utiliza la ley del menor esfuerzo. Por ejemplo, en el apellido aimara fonema aimara postvelar /q/ es reemplazado por el fonema velar / k/ por lo que se escucha Kolke en lugar de Qullqi, lo que da cuenta que la realización de los sonidos post-velares del aimara se tornan más difíciles para el aparato fonador del castellano hablante. Por tal motivo, se recurre al sonido próximo del sistema fonológico y que requiere de menor esfuerzo (Coaquira, 2014). Los apellidos aimaras en su mayoría son difíciles de pronunciar, por ese motivo, 
Transformaciones fonológicas, morfológicas y semánticas de los apellidos aimaras... Milusca T. Arocutipa, Elizabeth Castrillo, Carmen J. Mamani y Vilma Ramos

muchas personas llegan a castellanizar el apellido Qullqi por Kolke facilitando así la pronunciación y recurriendo a la ley del menor esfuerzo.

Según Condori (2007) el apellido Qullqi con el pasar del tiempo llegó a modificarse, algunos optaron por la castellanización llegando a convertirse en «Colque»; mientras que otros prefirieron cambiar por su equivalencia en castellano «Plata» y otros se inclinaron aún más por adicionar sufijos a la raíz del apellido traducido al castellano, quedando como «Platero». Por lo mencionado, el autor nos da a entender que el apellido Qullqi de origen aimara fue castellanizado, modificado y adicionado sufijos a la raíz, obteniendo así tres apellidos parecidos a la raíz, pero con significados distintos. Para mostrar una evidencia de la evolución de dicho apellido, Albo, citado por Yapu (2005), muestra un cuadro sobre la forma y origen de los apellidos aimaras, dicha información se presenta en la siguiente tabla:

Tabla 2. Transformación y observaciones del apellido Colque

\begin{tabular}{|c|c|c|c|c|}
\hline \multicolumn{3}{|c|}{ Apellido aymara original } & \multirow[t]{2}{*}{ Apellidos transformados } & \multirow{2}{*}{ Observaciones } \\
\hline $\begin{array}{l}\text { Forma } \\
\text { regular }\end{array}$ & $\begin{array}{l}\text { Origen } \\
\text { fonético }\end{array}$ & $\begin{array}{l}\text { Traducción } \\
\text { literal }\end{array}$ & & \\
\hline $\begin{array}{l}\text { Aru } \\
\text { Colque } \\
\text { Pajsi } \\
\text { Mamani } \\
\text { Condori } \\
\text { Catari } \\
\text { Coila }\end{array}$ & $\begin{array}{l}\text { aru } \\
\text { qulqi } \\
\text { phaxsi } \\
\text { mamani } \\
\text { kunturi } \\
\text { katari } \\
\text { quyla }\end{array}$ & $\begin{array}{l}\text { palabra } \\
\text { plata } \\
\text { luna } \\
\text { halcón } \\
\text { cóndor } \\
\text { víbora } \\
?\end{array}$ & $\begin{array}{l}\text { Palabra } \\
\text { Plata } \\
\text { Luna } \\
\text { Aguilar, Alcón, Halcón } \\
\text { Aguilar, Condorena } \\
\text { Catarina } \\
\text { Cuela, Cuellar } \\
\text { Cúter, Gutiérrez } \\
\text { Chuquimia } \\
\text { Velasco } \\
\text { Moller, Moeller, Muller } \\
\text { Gisbert, Quisbert }\end{array}$ & $\begin{array}{l}\text { Traducción literal } \\
\text { Traducción literal } \\
\text { Traducción literal } \\
\text { Traducción aproximada y } \\
\text { literal } \\
\text { Traducción aproximada } \\
\text { Traducción fonética } \\
\text { Traducción fonética } \\
\text { Traducción fonética } \\
\text { Traducción fonética } \\
\text { Traducción fonética } \\
\text { Traducción fonética a } \\
\text { apellido alemán } \\
\text { Aproximación fonética a } \\
\text { apellido }\end{array}$ \\
\hline
\end{tabular}

Fuente. Albo, citado por Yapu (2005, p.36).

En conclusión, el apellido Qullqi es proveniente de la lengua aimara, el cual con el paso del tiempo fue cambiando y a nivel fonológico y semántico. Las causas de la transformación del significado original del apellido mencionado anteriormente, fue la castellanización y la sustitución de fonemas de la lengua aimara por 
Transformaciones fonológicas, morfológicas y semánticas de los apellidos aimaras... Milusca T. Arocutipa, Elizabeth Castrillo, Carmen J. Mamani y Vilma Ramos

fonemas de la lengua castellana; asimismo, el racismo que sufrieron los portadores de este apellido.

\subsection{Mamani}

El apellido Mamani se extendió en todo el Collasuyo hacia las comunidades rurales, urbanas y países de habla aimara; al respecto, Condori (2007) menciona que el apellido «Mamani en la cultura aymara es totémico por sus creencias culturales ya que son evocados como símbolos sagrados en sus prácticas religiosas» (p. 113). En ese entender, el autor considera que el apellido Mamani permanece desde tiempos antiguos, ya que hace referencia a hombres de águilas que destacaban por su lucha y la reivindicación. Este apellido aparte de ser un nombre de ave, también tiene otra función toponímica de nombres en comunidades: Mamaniri, Mamanica, Marquiri entre otros. En este sentido, el apellido mantiene su origen, y no perdió su originalidad; tampoco su

contenido y significado, [...] palabra Mamani es un morfema base o palabra [...] que significa Halcón, Águila. Sin embargo, con la evolución del tiempo este apellido Mamani ha sufrido cambios por el fenómeno diglosia, inicialmente unos optaron por la traducción al castellano. (Condori, 2007, p. 113)

Los autores hacen referencia que el apellido tuvo cambios en el transcurso del tiempo, debido a que llegó a castellanizarse.

Cerrón-Palomino, citado en Yapu (2005), afirma que el «origen de MAMANI es WAMAN, que posteriormente se le agrega al final el fonema vocálico /i/ llegando a ser WAMANI; luego pasa a ser MAMANI, la ' $W$ ' fue reemplazada por ' $M$ '» (p. 175). El autor da cuenta de la sustitución del fonema $/ w /$ por $/ \mathrm{m} /$ sin embargo, esto no ha variado en su significado.

Tabla 3. Transformación del apellido Mamani

\begin{tabular}{l|l|l|l|}
\hline Apellido original & $\begin{array}{l}\text { Significado o } \\
\text { aproximación }\end{array}$ & \multicolumn{2}{|c|}{ Apellido resultado de la sustitución } \\
\cline { 2 - 3 } & $\begin{array}{l}\text { Cambio semántico } \\
\text { Halcón o águila } \\
\text { El creador }\end{array}$ & Aguilar & Maydana, Morales \\
\hline Mamani & & \\
\hline
\end{tabular}

Fuente. Condori (2007). 
Transformaciones fonológicas, morfológicas y semánticas de los apellidos aimaras... Milusca T. Arocutipa, Elizabeth Castrillo, Carmen J. Mamani y Vilma Ramos

Condori (2007) clasifica en tres sustituciones al apellido Mamani: fonológica, semántica y léxica:

a. La sustitución fonológica: la fonologización de los fonemas aimaras no tuvo ninguna variación en el castellano. En cuanto, a los fonemas se realizan tal cual, porque ambos $/ w / y / m /$ tienen el mismo sonido que están presente.

b. La sustitución semántica: el apellido Mamani en el transcurso del tiempo fue cambiado por su equivalencia de Halcón y Aguilar, que forma parte del mismo campo semántico, relacionándose con el significado. Además, tiene el mismo significado tanto en castellano como en aimara.

c. La sustitución léxica: el apellido es reemplazado por apellidos foráneos como Maydana, Morales, entre otros. Esta sustitución léxica de los apellidos mencionados se puede percibir que no tiene nada en común con el apellido Mamani.

En síntesis, el apellido Mamani en el transcurso del tiempo con los cambios semánticos y lexicales quedan de esta manera «Mamani > Aguilar > Maidana».

\subsection{Qhispi - Quispe}

El apellido Quispe es bastante usado en las zonas altiplánicas de nuestro país, ya que muchas familias son portadores de la misma. Este apellido es una palabra de la lengua aimara y desde el estudio de la antroponimia pertenece a la categoría de antropónimos simples, las mismas que se caracterizan porque son una palabra base en una lengua. Según Gálvez y Olaechea (2005), «estos antropónimos tienen, posiblemente, origen en nombres de plantas, animales, características u objetos representativos, así como en la procedencia de la persona» (p. 44). Por lo señalado, es posible decir que los antropónimos simples surgen de nombres propios de los elementos que se encuentran alrededor de la naturaleza, ya sean animales, plantas, lugares incluso cualidades y características de los mismos.

En esa línea, el apellido Qhispi de la lengua aimara significa 'brillante' en castellano. Por lo tanto, se puede decir que Qhispi es una palabra base que se originó para representar una característica de un objeto. Sin embargo, es preciso mencionar que quienes portaban dicho apellido sufrían discriminación, lo cual los encaminaba a la alienación. Según Choque (2005): 
Transformaciones fonológicas, morfológicas y semánticas de los apellidos aimaras... Milusca T. Arocutipa, Elizabeth Castrillo, Carmen J. Mamani y Vilma Ramos

La alienación va más allá, los apellidos originarios son cambiados o castellanizados, por ejemplo, de «Yana Qaqa» a «Monte Negro», de «Pampa Wasi» a «Casa de la Pradera», de «Yuraq Q’asa» a «Abra Blanca», etc. La alienación va más allá, los apellidos originarios también son cambiados o castellanizados, de «Qhispi» a «Quispe» y luego a «Quisbert», de «Willka» a «Villca» y luego a «Vilchez», de «Mamani» a «Magne», de «Wanka» a «Cuenca», de «Mamani» a «Magne», de «Wanka» a «Cuenca», de «Kunturi» a «Condori», y luego a «Condorcet»; y, así sucesivamente. (p. 19)

Lo señalado por el autor, da cuenta que la alienación influye también en las decisiones de las personas para cambiar sus apellidos originarios. Por ejemplo, en un primer momento, optan por castellanizar y posteriormentellegan a cambiarlas agregándoles sufijos, así como sucede con el apellido Qhispi, primero lo castellanizan a Quispe y posteriormente agregan sufijos y logran convertirlo en Quisbert, así como se muestra en la siguiente tabla:

Tabla 4. Aproximación y resultado de la sustitución del apellido Quispe

\begin{tabular}{l|l|l|l|}
\hline Apellido original & $\begin{array}{l}\text { Significado o } \\
\text { aproximación }\end{array}$ & \multicolumn{2}{|c}{ Apellido resultado de la sustitución } \\
\cline { 4 - 4 } & Cambio semántico & Cambio semántico \\
\hline Qhispi & Brillante & Quispe & Quisbert \\
\hline
\end{tabular}

Fuente. Elaboración propia.

Al respecto, Condori (2007) da a conocer que el apellido Qhispi sufrió por un lado un cambio fonológico y por otro lado un cambio semántico de la siguiente manera:

a) En la sustitución fonológica, los fonemas que sufrieron alteraciones son: el fonema /qh/oclusivo, aspirado, postvelar, del aymara son sustituidos por el fonema / $k$ / oclusivo, simple, velar, de esta manera sufrió una velarización, además de una simplificación del postvelar. El fonema vocálico /i/ anterior, alta, pasa como /e/ vocal anterior, media.

b) En la sustitución semántica, el apellido aimara Qhispi fue cambiado por su similar en castellano Espejo. (p. 133)

Por lo señalado, es posible decir que el apellido Qhispi sufrió una transformación fonológica, debido a la sustitución de los fonemas aimaras $/ q h /$ por $/ k /$, de 
Transformaciones fonológicas, morfológicas y semánticas de los apellidos aimaras... Milusca T. Arocutipa, Elizabeth Castrillo, Carmen J. Mamani y Vilma Ramos

igual manera /i/ por /e/ vocal media, por lo que se llega a escuchar Kispe y desde el sistema fonológico castellano se escucha Quispe. De la misma forma, semánticamente también el apellido Qhispi sufrió una transformación debido a que fue reemplazado por la traducción del castellano, se sustituyó de Qhispi a Espejo. Lo anterior, da cuenta del proceso de transformación fonológica y semántica que sufrió el apellido aimara Qhispi.

\subsection{Ch'uqi - Choque}

El apellido Ch'uqi es uno de los apellidos comunes que posee una gran cantidad de población en las zonas donde se habla la lengua aimara, en el altiplano, el cual:

Antiguamente significaba oro, metal precioso. Después, se asignó el nombre de patata o papa, del mismo modo, se dice al recado en conocimiento que está crudo o poco cocido. La característica de los portadores del apellido Ch’uqi se les conoce como personas afables y unificadores. (Condori, 2007, p. 121)

Por lo mencionado, es posible decir que el apellido Ch'uqi posee diversos significados desde un metal precioso como el oro, hasta el nombre de un tubérculo como la papa o la atribución de un alimento crudo.

En esa misma línea, la RENIEC (2018) en su libro Tesoro de nombre aimara señala que Chuqi significa «Oro, cosa de gran valor. Persona de gran valor, deseada por todos, buscada y reconocida por su gran valor y calidad humana» (p. 133). Por lo que señala la RENIEC, se puede mencionar que Chuqi significa valioso, ya sea referido a un metal o persona. No obstante, en la actualidad las personas portadoras de dicho apellido la han transformado al castellanizar de Chuqi a Choque. Al respecto, Yapu (2005) menciona que «El apellido aymara 'Ch'uqi' pasó al castellano como 'Choque', en el idioma de origen el apellido significa 'papa, crudo, oro', ahora en castellano significa 'coalición, golpe'» (p. 124). De esta manera, se evidencia la transformación que tuvo a nivel fonológico. Asimismo, da cuenta del cambio del significado, así como se muestra en la siguiente tabla:

Tabla 5. Aproximación y resultado de la sustitución del apellido Choque

\begin{tabular}{|c|c|c|}
\hline \multirow{2}{*}{ Apellido Original } & \multirow{2}{*}{$\begin{array}{l}\text { Significado o } \\
\text { aproximación }\end{array}$} & Apellido resultante de la sustitución \\
\hline & & Cambio fonológico \\
\hline Ch’uqi & Oro. Papa. Crudo & Ch'uqi $>$ Choque \\
\hline
\end{tabular}

Fuente. Condori (2007, p. 121). 
Transformaciones fonológicas, morfológicas y semánticas de los apellidos aimaras... Milusca T. Arocutipa, Elizabeth Castrillo, Carmen J. Mamani y Vilma Ramos

Como se muestra en el cuadro, el apellido Chuqi sufre una transformación fonológica al sustituir el fonema /ch'/ del aimara por el fonema /ch/ del castellano, asimismo al sustituir los fonemas vocálicos /u/ e /i/ del aimara por los fonemas vocálicos /o/ y /e/.

A nivel semántico, el apellido aimara Chuqi también llega a sufrir una transformación, ya que llega a ser sustituido por Paz, así como da a conocer Yapu (2005) en el siguiente tabla:

Tabla 6. Castellanización y sustitución léxica del apellido Choque

\begin{tabular}{|l|l|}
\hline Apellido Castellanizado & $\begin{array}{l}\text { Apellido resultante de la } \\
\text { sustitución léxica }\end{array}$ \\
\hline Choque & $\mathrm{Paz}$ \\
\hline
\end{tabular}

Fuente. Yapu (2005).

Como se puede observar en el cuadro, el apellido Ch'uqi, después de sufrir una transformación al ser castellanizado, sufre también una transformación semántica, ya que es sustituida por el apellido Paz; el cual, no guarda relación con ninguno de los significados de Ch'uqi en la lengua aimara. Lo señalado, da cuenta de la transformación a nivel fonológico y a nivel semántico del apellido Ch’uqi.

\subsection{Mallku, equivalente de Cóndor}

La palabra Mallku tiene un significado en la lengua aimara, según Castro (2015) señala en el Diccionario ilustrado de la lengua aymara que significa 'autoridad'. En la actualidad, en Bolivia existe una organización de autoridades comunales aimaras llamado Mallkus, el cual se basa en la idea moral de que «los mallkus cuidan la tierra» (Funko, 2015, p. 103). Por lo señalado, es posible decir que mallku significa autoridad que cuida la tierra, haciendo referencia al cuidado de la naturaleza.

Para el RENIEC (2018), la palabra mallku también adquiere otro significado que es el siguiente: 
Transformaciones fonológicas, morfológicas y semánticas de los apellidos aimaras... Milusca T. Arocutipa, Elizabeth Castrillo, Carmen J. Mamani y Vilma Ramos

Tabla 7. Significado de Mallku

\begin{tabular}{l|l|l}
\hline Nombre & Significado en la lengua & Significado en el nombre \\
\hline Mallku & $\begin{array}{l}\text { Cóndor, } \\
\text { el cóndor } \\
\text { mitológico. }\end{array}$ & $\begin{array}{l}\text { La fuerza de las montañas. } \\
\text { Dios tutelar del aire y las } \\
\text { alturas. }\end{array}$ \\
\hline
\end{tabular}

Fuente. RENIEC (2018, p. 138).

Lo anterior hace ver que el significado en aimara hace referencia a un ave andino, el cóndor, quien a su vez también es una deidad desde la cosmovisión andina, el cual es compartido por las culturas aimara y quechua. Esta denominación del ave cóndor en la lengua quechua es kuntur, cuyos portadores de este apellido actualmente apellidan Condori.

El apellido Condori, en la actualidad es otro de los apellidos con mayor población de portadores al igual que el apellido Mamani a nivel de la región altiplánica de nuestro país. Sin embargo, es probable que el apellido Condori fue aimarizado por la población aimara hablante, así como señala RENIEC (2018):

El apellido Condori es un quechuismo aimarizado. Como se sabe, el aimara no permite palabras que terminan en consonante. Al prestarse una con dichas características, le añade una vocal al final. De este modo, la palabra kuntur, cóndor, el mayor buitre andino, fue ligeramente modificada con una vocal final, y luego sufrió el ajuste fonológico al español. (p. 120)

Lo anterior, da cuenta del proceso de transformación que tuvo el apellido quechua Kuntur para posteriormente ser aimarizado agregando así el fonema vocálico /i/, quedando como Kunturi para ser usado por la población aimara, puesto que el ave cóndor tiene su propia denominación en la lengua aimara que es mallku. Por lo tanto, se puede afirmar que el apellido Kunturi tiene origen en la lengua quechua, pero que fue adoptado por la lengua aimara.

El apellido Kunturi significaría el representante de los dioses, enviado de los espíritus. Cóndor, ave rapaz diurna, tótem de lugar o de la casa (Condori, 2007). Asimismo, Coaquira (2014) en su artículo llamado «Apellidos aymaras desde el enfoque lingüístico» indica que Kunturi significaría como 'representante de los dioses'. Por lo tanto, es un apellido que significa cóndor, ave sagrado de la cosmovisión andina.

236 Lengua y Sociedad. Revista de Lingüística Teórica y Aplicada 
Transformaciones fonológicas, morfológicas y semánticas de los apellidos aimaras... Milusca T. Arocutipa, Elizabeth Castrillo, Carmen J. Mamani y Vilma Ramos

Generalmente, quienes cambiaron su apellido fueron los migrantes; primero, por estatus, querían formar parte del ejército, policía o funcionarios de esta manera tendrían la oportunidad laboral y acceder a diferentes espacios (Pozo, 2015). En esa línea. en la actualidad el apellido Kunturi fue cambiado por sus portadores «muchos Kunturi originarios del lugar optaron por cambiar su apellido, inicialmente por la refonimización a Condori, luego por la omisión o adición de algunos elementos como: Conde, Condorset, Condarco y Contreras» (Condori, 2007, p. 118). Lo señalado por Condori, da cuenta de la transformación fonológica y morfológica que sufrió el apellido Kunturi, lo cual se resume en el siguiente tabla:

Tabla 8. Aproximación y sustitución del apellido Condori

\begin{tabular}{|l|l|l|l|l|}
\hline $\begin{array}{l}\text { Apellido } \\
\text { original }\end{array}$ & $\begin{array}{l}\text { Significado o } \\
\text { aproximación }\end{array}$ & $\begin{array}{l}\text { Apellido resultado de la sustitución } \\
\text { Cambio } \\
\text { fonológico }\end{array}$ & $\begin{array}{l}\text { Cambio } \\
\text { morfológico }\end{array}$ & Cambio léxico \\
\hline Kunturi & $\begin{array}{l}\text { Cóndor. Rep. } \\
\text { de dioses }\end{array}$ & $\begin{array}{l}\text { Kunturi }> \\
\text { Condori }\end{array}$ & $\begin{array}{l}\text { Condorset } \\
\text { Condorena }\end{array}$ & Contreras \\
\hline
\end{tabular}

Fuente. Condori (2007, p. 119).

Como se puede observar en el cuadro, el apellido Kunturi sufrió una transformación a nivel fonológico, debido a que los fonemas $/ k / / u / / t / e / i /$ fueron sustituidos por los fonemas del castellano $/ \mathrm{c} / / \mathrm{u} / / \mathrm{d} / / \mathrm{u} / \mathrm{e} / \mathrm{i} /$. Mientras que, a nivel morfológico, la raíz del apellido Kunturi que fue kuntur al ser castellanizada Cóndor fue sufriendo transformaciones, ya que se le añadió diversos sufijos como -set y -ena, los cuales fueron alterando totalmente en significado original de dicho apellido.

\section{Conclusiones}

La antroponimia se encarga de realizar estudios de los nombres de las personas o también llamado ciencia de los nombres (Condori, 2007); por ello, en este artículo se realizó análisis de cinco apellidos aimaras: Mamani, Qhispi, Qullqi, Ch'uqi y Kunturi, puesto que son los apellidos más comunes a nivel nacional, los cuales con el pasar de los años desde la lingüística sufrieron transformaciones fonológicas, morfológicas y semánticas, lo cual repercutió en la pérdida de sus significados originales. 
Transformaciones fonológicas, morfológicas y semánticas de los apellidos aimaras... Milusca T. Arocutipa, Elizabeth Castrillo, Carmen J. Mamani y Vilma Ramos

En cuanto a las transformaciones fonológicas de los apellidos aimaras, se ha podido constatar que éstos sufrieron sustituciones de fonemas aimaras aspiradas a fonemas simples, como el cambio del fonema /qh/ por el fonema $/ \mathrm{k} /$, mientras que en otros apellidos, los fonemas han sido reemplazadas por fonemas de la lengua castellana, como los fonemas vocálicos fuertes a medias, por ejemplo /i/ por /e/ y /u/ por / o/. Respecto a los cambios morfológicos, se logró dar cuenta que en el apellido aimara se agregó un fonema vocálico, como en el caso del apellido Waman que se le agregó /i/ quedando como Wamani y posteriormente como Mamani. Finalmente, en cuanto a los cambios semánticos se pudo dar cuenta que algunos apellidos aimaras fueron sustituidos por las traducciones del castellano como el apellido Qhispi que fue reemplazado por Espejo.

Realizar este artículo fue imprescindible, ya que ayuda a descifrar los apellidos aimaras desde sus orígenes y significados. Por ello, es importante seguir realizando investigaciones acerca de otros apellidos aimaras, debido a que muchos fueron cambiados por traducciones del castellano, esto permitirá conocer y fortalecer nuestra identidad cultural.

\section{Referencias bibliográficas}

Aranya, J. (2009). La valoración del componente fonológico en niños y niñas que inician el proceso de lecto-escritura. Káñina, 33(2), 33-41. https://www.redalyc. org/pdf/442/44248785003.pdf

Bustos, E. (1998). Modelos morfológicos y cambio morfológico. Revista de Filología Románica, 15, 35.49. https://www.researchgate.net/publication/27574592_ Modelos_morfologicos_y_cambio_morfologico

Castro, E. (2015). Diccionario ilustrado de la lengua aymara. Ministerio de Educación de Chile. http://peib.mineduc.cl/wp-content/uploads/2019/10/ diccionario_aymara_145_230.pdf

Choque, C. (2005). La еIB entre los quechuas: testimonio de parte (1990-1994). PINSEIB; PROEIB Andes; Plural. http://bvirtual.proeibandes.org/publicaciones/ publicaciones/17.pdf

Coaquira, J. (2014). Apellidos aymaras desde el enfoque lingüístico. https:// kupdf.net/download/apellidos-aymaras-desde-el-enfoque-linguisticopdf_5ao1e441e2b6f5031do82897_pdf

Condori, L (2007). Estudio diglósico de la antroponimia en la cultura aymara [Tesis de licenciatura, Universidad Mayor de San Andrés]. Repositorio 
Transformaciones fonológicas, morfológicas y semánticas de los apellidos aimaras... Milusca T. Arocutipa, Elizabeth Castrillo, Carmen J. Mamani y Vilma Ramos

Institucional de la Universidad Mayor de San Andrés. https://repositorio. umsa.bo/handle/123456789/16857

Funko, A. (2015). Los mallkus de uraqita: la obligación que legitima la posesión de la tierra. Integra Educativa, 7(4), 103-140. http://www.scielo.org.bo/pdf/ rieiii/v8n1/v8n1_ao6.pdf

Gálvez, I y Olaechea C. (2005). Onomástica en el área norcentral del Perú, valle de Pativilca Sur: topónimos y antropónimos. Fabla, 3(3), 31-52. https://sisbib. unmsm.edu.pe/bibvirtualdata/publicaciones/fabla/2005_no3/ao2.pdf

Gómez, M., Pierre, J. y Alzate, M. (2010). Cómo hacer tesis de maestría y doctorado. Investigación, escritura y publicación. Ecoe. https://corladancash.com/ wp-content/uploads/2019/08/55-Como-hacer-tesis-de-maestria-y-doctorado-GOMEZ.pdf

Hernández, R., Fernández, C. y Baptista, M. (2014). Metodología de la investigación. Mcgraw-Hill; Interamericana. https://www.uca.ac.cr/wp-content/ uploads/2017/10/Investigacion.pdf

Jacinto, E. (2009). Estudio del sistema de denominación antroponímica de la cultura asháninka [Tesis de licenciatura, Universidad Nacional Mayor de San Marcos]. Cybertesis UnMsm. http://cybertesis.unmsm.edu.pe/bitstream/ handle/20.500.12672/954/Jacinto_sp.pdf;jsessionid=983AoA561AD7A4FCDAС 3500 BF4АВС 9 BC? sequence $=1$

Laura, S. (2014). Estudio léxico-semántico de la toponimia aymara en la población de Compi-Tauca, de la provincia Omasuyos del departamento de La Paz [Tesina de técnico superior, Universidad Mayor de San Andrés]. Repositorio Institucional de la Universidad Mayor de San Andrés. https://repositorio.umsa. bo/bitstream/handle/123456789/16132/T-3445.pdf? sequence $=1$ \&isAllowed $=y$ Lovón, M. (2019). Las palabras compuestas en el aimara de Conima. Lexis, 43(1), 55-85. https://doi.org/10.1880o/lexis.201901.002

Orellana, A. (2007). Morfología nominal del aimara de Zepita [Tesis de maestría, Universidad Nacional Mayor de San Marcos]. CORE. https://core.ac.uk/download/pdf/323346213.pdf

Pozo, E. (2015). La promesa de lo aimara: La «Unión de Comunidades Aymaras» (UNCA) y la formación del discurso identitario reivindicativo en el sur de Puno [Tesis de maestría, Pontificia Universidad Católica del Perú]. Repositorio Institucional de la Pontificia Universidad Católica del Perú. http://tesis.pucp. edu.pe/repositorio/bitstream/handle/20.500.12404/5916/pozo_buleje_erik_ promesa.pdf? sequence $=1$ \&isallowed $=y$ 
Transformaciones fonológicas, morfológicas y semánticas de los apellidos aimaras... Milusca T. Arocutipa, Elizabeth Castrillo, Carmen J. Mamani y Vilma Ramos

Pérez-Ruiz, M. (2000). Nacido indio, siempre indio. Discriminación y racismo en Bolivia. Nueva Antropología, 17(58), 73-87. https://www.redalyc.org/ pdf/159/15905804.pdf

RENIEC. (2012). Introducción a un tesoro de nombres quechuas en Apurímac. Terra Nuova; Apurimac Onlus; Registro Nacional de Identificación y Estado Civil. http://www.terranuova.org.pe/portal/sites/default/files/Introduccion\%20a\%20 un\%2otesoro\%2ode\%2onombres\%2oquechuas.pdf

RENIEC. (2015). Tesoro de nombres Jaqaru. Shumay shutxkuna jaqarna. Registro Nacional de Identificación y Estado Civil (RENIEC). https://www.reniec.gob.pe/ portal/html/registro-civil-bilingue/portalrcb2016/4-difusion/9-publicaciones/ tesoro-nombres-jaqaru.pdf

RENIEC. (2018). Tesoro de nombre Aimara. Registro Nacional de Identificación y Estado Civil. https://www.iidh.ed.cr/capel/media/1519/tesoro-de-nombres-aimara.pdf

Sonco, N. (2015). Análisis contrastivo del sistema fonológico a nivel consonántico entre el idioma aymara e inglés [Tesina de técnico superior, Universidad Mayor de San Andrés]. https://repositorio.umsa.bo/bitstream/handle/123456789/16131/T-3523.pdf? sequence $=1$ \&isAllowed $=\mathrm{y}$

Yapu, N. (2005). Tipología de cambio de apellidos aymaras en la Paz (Estudio documental) [Tesis de grado, Universidad Mayor de San Andrés]. Repositorio Institucional de la Universidad Mayor de San Andrés. https:// repositorio.umsa. bo/bitstream/handle/123456789/15934/T-1180.

240 Lengua y Sociedad. Revista de Lingüística Teórica y Aplicada 
Transformaciones fonológicas, morfológicas y semánticas de los apellidos aimaras... Milusca T. Arocutipa, Elizabeth Castrillo, Carmen J. Mamani y Vilma Ramos

\section{Trayectoria académica de los autores}

\section{Milusca Teobalda Arocutipa Saira}

Natural del distrito de Acora, región Puno, es licenciada en Educación Inicial Intercultural Bilingüe de la Universidad Peruana Cayetano Heredia - Lima. Actualmente es docente de Educación Básica Alternativa (CEBA).

\section{Ruth Elizabeth Castrillo Yavar}

Natural del distrito de Puno, bachiller en Lengua, Literatura Psicología y Filosofía por la Universidad Nacional del Altiplano Puno. Actualmente se encuentra trabajando en la ciudad de Juliaca.

\section{Carmen Justina Mamani Paucar}

Natural del distrito de Santa Rosa, Melgar, Puno; quechua hablante de la variedad Cusco-Collao. Asimismo, es licenciada en Educación Primaria Intercultural Bilingüe por la Universidad Peruana Cayetano Heredia, miembro de la revista digital quechua Khipu y del Club de Poetas Originarios del Perú.

\section{Vilma Ramos Maque}

Originaria del distrito de Macusani, Carabaya, Puno. Licenciada en la carrera de Educación Primaria Intercultural Bilingüe por la Universidad Peruana Cayetano Heredia - Lima, traductora e intérprete de lengua quechua collao por el MINCUL y actualmente es estudiante de maestría de Lingüística Andina y Educación de la Universidad Nacional del Altiplano, Puno. 